\title{
Laparoscopic Treatment of Vesicovaginal Fistula
}

\author{
Marcos Tobias-Machado', Pablo Aloisio Lima Mattos¹, Pedro Romanelli de Castro², Ricardo Hissashi \\ Nishimoto ${ }^{3}$, Antonio Carlos Lima Pompeo ${ }^{1}$
}

${ }^{1}$ Section of Minimally Invasive Surgery, Department of Urology, ABC Medical School, Santo André, São Paulo; ${ }^{2}$ Section of Minimally Invasive Surgery, Mater Dei Hospital, Belo Horizonte, Minas Gerais and ${ }^{3}$ Department of Urology, Minas Gerais Hospital Foundation, MG, Brazil

\begin{abstract}
Introduction: Vesicovaginal fistula is a rare disease with great impact for the patients. Laparoscopic repair can be an interesting option in selected cases with goods results but few experience is reported.

Objectives: Detailed demonstration of our laparoscopic vesicovaginal fistula repair technique. Initial results for ten patients are provided Methods: We treated all cases by the same technique. The surgical steps were: Patient positioning in Lloyd-Davis; Cystoscopy and implant of guide wire on fistula and ureteral catheters (that was removed after procedure); Transperitoneal access and 4 or 5 ports in V or W shape; Opening the bladder wall; Dissection between bladder and vagina for tension free repair; Fistula resection; Vagina repair with Vicryl 3-0; Bladder repair with Vicryl 3-0; Peritoneum/omentum interposition; Positioning 20 Fr urethral catheter.

Results: Mean age was 50 years. Mean number of fistulas was 1,2. The most common etiology was gynecologic surgery (7). Mean operative time was 2,5 (1,8-3,2) hours. Mean blood loss was 150 (100-200)mL. Complication rate was 10\% (one case of urinary infection treated conservatively). Mean hospital stay was 1,2 (1-2) days. Mean return to normal and activities was 20 (15-30) days. For nine patients mean sexual intercourse time was 3 (1-6) months. Success rate after 1 year was $90 \%$ (one case of recurrence in patient with previous radiotherapy). Mean follow-up was 36 (12-60) months.

Conclusions: Laparoscopic repair is feasible, reproducible and present all advantages of minimally invasive surgical procedure. Long term results are similar to conventional open approaches.
\end{abstract}

\section{ARTICLE INFO}

Available at: www.brazjurol.com.br/videos/may_june_2014/Tobias_Machado_435_436video.htm

Int Braz J Urol. 2014; 40 (Video \#9): 435-6

Submitted for publication:

March 30, 2014

Accepted after revision:

April 15, 2014
Correspondence address:

Pablo Aloisio Lima Mattos, MD Dona Veridiana, 115 / 13 São Paulo, SP, 01238-010, Brazil Fax: +55 11 3996-0045 E-mail: palmattos@hotmail.com 


\section{EDITORIAL COMMENT}

Vesicovaginal fistula is a potentially devastating complication. Repair of vesicovaginal fistula can prove challenging especially if the patient has received radiotherapy or the fistula is recurrent and/or large. Many vesicovaginal fistulae can be treated using a transvaginal technique $(1,2)$. The video by Tobias et al nicely depicts a laparoscopic technique that utilizes all the components of the

\section{REFERENCES}

1. Cohen BL, Gousse AE: Current techniques for vesicovaginal fistula repair: surgical pearls to optimize cure rate. Curr Urol Rep. 2007; 8: 413-8. open 0'Conor technique. Specifically, a transperitoneal approach to open the bladder, followed by excision of the fistula tract, dissection of the bladder off of the vagina with separate closures and omental interposition are used (3). This approach does require advanced laparoscopic skills. In their series with intermediate follow-up they are able to achieve excellent success rates. Their patients seemed to benefit from the laparoscopic approach as they were able to go home within 1-2 days.

Hubert Swana, MD Pediatric Urology Nemours Children's Hospital Orlando Orlando, FL, USA E-mail: hswana@nemours.org 